\title{
Is oxidative stress a factor in the pathogenesis of subacute thyroiditis?
}

\author{
Murat Dağdeviren (ํ) \\ Mustafa Altay ${ }^{1}$
}

${ }^{1}$ Department of Endocrinology and Metabolism, Keçiören Health Administration and Research Centre, University of Health Sciences, Ankara, Turkey

${ }^{2}$ Department of Medical Biochemistry, University of Yildırım Beyazıt, Ankara, Turkey

\begin{abstract}
Introduction: In a performed study, selenium levels were detected to be lower in subacute thyroiditis (SAT). This outcome suggests that the oxidant-antioxidant system may play a role in the aetiopathogenesis of SAT, as is the case for many other diseases. The aim of the present study was to detect whether any association exists between SAT and oxidative stress, and to determine the factors of a possible association. Material and methods: Twenty-five patients who were newly diagnosed with SAT and 30 healthy volunteers were enrolled in the study. Total antioxidant status (TAS), total oxidant status (TOS), oxidative stress index (OSI), paraoxonase 1 (PON1), and dynamic thiol/disulphide [native thiol (NT), total thiol (TT), disulphide (DS), DS/TT, DS/NT, and NT/TT] levels of the participants were evaluated.

Results: Total thiol and NT levels were found to be lower in the SAT group $(\mathrm{p}<0.001)$. DS/NT and DS/TT levels were significantly higher in the patients with SAT, whereas NT/TT levels were lower $(\mathrm{p}<0.05)$. A negative correlation was detected between C-reactive protein (CRP) and TAS, whereas a positive correlation was detected between CRP and OSI. There was a negative association between TSH and TAS only in the partial correlation analysis by adjusting for age, white blood cell count, neutrophil (Neu) level, CRP, and erythrocyte sedimentation rate $(\mathrm{ESR})(\mathrm{r}=-0.481, \mathrm{p}=0.043)$.

Conclusions: Thiol levels are significantly decreased and thiol/disulphide homoeostasis is disrupted in patients with SAT. The present study has presented for the first time that there may be an association between SAT and oxidative stress. (Endokrynol Pol 2022; 73 (1): 64-70)

Key words: subacute thyroiditis; pathogenesis; oxidative stress; inflammation; dynamic thiol/disulphide
\end{abstract}

\section{Introduction}

Subacute thyroiditis (SAT) is a condition presenting with local or generalized inflammation of the thyroid gland. The main symptoms include fever appearing with inflammation, pain, and tenderness of the neck. Although the aetiology is not clear, there is a history of upper respiratory tract infection, which appears 2 to 8 weeks before thyroiditis. Therefore, SAT is thought to be related to a viral infection or post-viral inflammation. Autoimmunity does not play a primary role in the progress of the disease. However, a strong association with HLA-B35 molecules was revealed in many ethnic groups. Therefore, some authors suggest that SAT may develop due to transmission of viral infection in individuals with a genetic tendency [1].

Oxidative stress appears as a result of the over-expression of free oxygen radicals and the deficiency of antioxidant defence systems. The reactive oxygen radicals that appear cause tissue damage [2,3]. There are many molecules reflecting the oxidant and antioxi- dant status of an organism. For instance, total oxidant status (TOS) and total antioxidant status (TAS) reflect the general condition of oxidative stress in the body. Paraoxonase 1 (PON1) is an enzyme that protects low-density lipoprotein (LDL) against oxidative damage and is closely related to high-density lipoprotein (HDL) [4]. Thiols are the compounds including sulfhydryl groups connected to carbon atoms. Total thiol (TT) is an antioxidant that provides protection against tissue and cell damage by reacting with free oxygen radicals. Oxygen radicals oxidize the thiol groups of the proteins consisting of amino acids including sulphur and create disulphide (DS) bonds. Such disulphide bonds are the earliest indicators of protein oxidation, and these bonds are reversible. Reduction of reversible disulphide bonds provides the protection of dynamic thiol/disulphide homeostasis [5-8]. Homoeostasis of dynamic thiol/disulphide plays a key role in cellular activities including antioxidant defence, signal transmission, detoxification, cell proliferation, and apoptosis. When thiol/disulphide homoeostasis is disrupted as a result 
of oxidative stress and the balance slides towards disulphide, functional disorders appear in the aforesaid cellular activities. Therefore, abnormal thiol/disulphide homoeostasis is expected to be associated with the aetiopathogenesis of many acute or chronic diseases [9].

The most important mechanisms in aetiopathogenesis appear to be genetic predisposition and viral infections; however, the exact cause has not been revealed yet. New hypotheses and studies are therefore needed to clarify the causes and risk factors for SAT. In a previous study performed within this scope, selenium levels were detected to be lower [10]. This outcome suggests that the oxidant-antioxidant system may play a role in the aetiopathogenesis of SAT, as is the case for many other diseases.

However, to the best of our knowledge, no previous study has indicated the association between SAT and oxidative stress directly. We therefore planned the present study to detect whether any association exists between SAT and oxidative stress and the determinant factors that affect such a possible association.

\section{Materials and methods}

The study was approved Ethics Committee of Clinical Research at Keçiören Education and Research Hospital, University of Health Sciences (Project No: 2012-KAEK-15/1701) before the onset of the study. The study was carried out in accordance with the Helsinki Declaration, and verbal and written consent was obtained from all participants.

Forty-three patients who had been referred as endocrinology outpatients between 15 June 2018 and 1 May 2019, and had been diagnosed with SAT were enrolled in the study. The exclusion criteria were diabetes mellitus, hypertension, cardiovascular or cerebrovascular disease, acute or chronic kidney or liver disease, any findings for acute or chronic infection, any rheumatologic disease or malignancy, and the use of any antioxidants, lipidlowering drugs, vitamin supplements, tobacco, or alcohol. A tota of 18 individuals with at least one exclusion criterion were excluded. The present study was thus completed with 25 patients who were newly diagnosed and had not received any treatment, as well as 30 healthy volunteers (Fig. 1).

SAT was diagnosed by history (pain on the anterior side of the neck, pain radiating to the ear, fever), physical exam (tenderness of the thyroid compartment), laboratory findings (thyrotoxicosis, increased erythrocyte sedimentation rate (ESR), C-reactive protein (CRP), and/or leukocyte values), and ultrasound findings (focal or diffuse heterogeneous, hypoechoic thyroid parenchyma with decreased vascularization) [1]

Venous blood samples of the participants were collected after a fasting period of at least 10 hours. Blood samples of the patient group were collected at the time of diagnosis, before the administration of any non-steroidal anti-inflammatory drug or steroid treatment. The blood samples were centrifuged at $1500 \mathrm{rpm}$ for 10 minutes, and serum and plasma were separated. The serum was transferred in Eppendorf tubes and stored at $-80^{\circ} \mathrm{C}$. All oxidative stress parameters were analysed once for the same serum sample.

The serum samples collected for CRP were analysed by the nephelometric method with a Beckman Coulter device (IMMAGE Immunochemistry Systems, Ireland). White blood cell (WBC) and neutrophil (Neu) counts were analysed with an LH 750 Analyzer (Beckman Coulter, UK). Thyroid-stimulating hormone (TSH) (normal range: 0.6-4 mIU/mL), free T4 (fT4) (normal range: 0.8-1.7 ng/ $\mathrm{dL}$ ), and free T3 (fT3) (normal range: $2-3.9 \mathrm{ng} / \mathrm{dL}$ ) were measured by electroluminescence immunoassay method with a Cobas e 601 analyser (Roche Diagnostics Corp., USA).

Serum TOS level was measured with a commercial kit (Rel Assay Diagnostics, Turkey; Ref. No: RL0024, Lot No: JE 14048Og) via colorimetric method with CV\% of 10 and linearity of $0-33.5 \mathrm{mmol} / \mathrm{L}$. The results are expressed in micromolar $\mathrm{H}_{2} \mathrm{O}_{2}$ equivalents per litre (mmol/ $\mathrm{L} \mathrm{H}_{2} \mathrm{O}_{2}$ equivalent/L) (normal range: 0-4 IU/mL).

Serum TAS level was measured with a commercial kit (Rel Assay Diagnostics, Turkey; Ref. No: RL0017, Lot No: JE 14042A) via colorimetric method with CV\% of 10 and linearity of $0-2.75 \mathrm{mmol} / \mathrm{L}$. The results are expressed in mmol Trolox equivalents/L $(\mathrm{mmol}$ Trolox equivalent/L).

The oxidative stress index (OSI) value was calculated according to the following formula: OSI (arbitrary unit) $=$ TOS $\left(\mathrm{mmol}_{2} \mathrm{O}_{2}\right.$ equivalent/L)/TAS (mmol Trolox equivalent/L) [4].

Serum PON1 level was measured with a commercial kit (Rel Assay Diagnostics, Turkey; Ref. No: RL0031, Lot No: JE14028P) via colorimetric method with CV\% of 5 and linearity of 0-750 U/L. PON1 activity was expressed as $\mathrm{U} / \mathrm{L}$ of serum.

Thiol/disulphide homeostasis tests were performed as described by Erel and Neselioglu [11]. Briefly, disulphide bonds were first reduced to form free functional thiol groups. Unused reducer sodium borohydride was consumed and removed with formaldehyde, including reduced and native thiol (NT); all thiol groups were determined after reaction with 5,5'-dithiobis-2-nitrobenzoic acid. The dynamic disulphide (DS) amount was calculated by determining half of the difference between the total thiol (TT) and the native thiol. After the native thiol, total thiol, and disulphide amounts were determined, the ratios of disulphide/total thiol (DS/ TT), native thiol/total thiol (NT/TT), and disulphide/native thiol (DS/NT) were calculated [11].

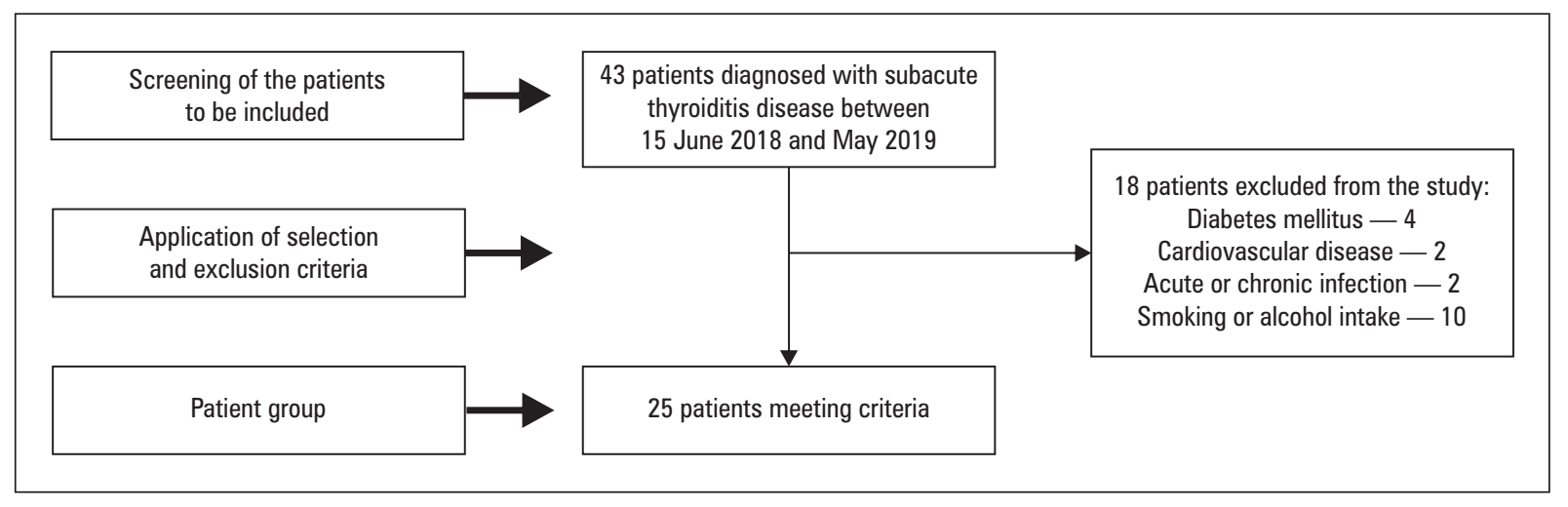

Figure 1. Determination of patient groups 


\section{Statistical analysis}

Data obtained from the present study were evaluated with the IBM SPSS Statistics 22 program (IBM Corp., USA). Kolmogorov-Smirnov/ Shapiro-Wilk tests and distributions of the data in histogram graphics were used to decide whether the data were distributed normally. Normally distributed data were expressed as mean \pm standard deviation, whereas non-normally distributed data were expressed as median (minimum-maximum). Categorical variables were expressed as percentages, and statistical analyses were conducted with the chi-square test. Student's t-test and the Mann-Whitney $\mathrm{U}$ test were used to assess the differences between the groups. Analysis of the associations between the variables was conducted with Spearman and Pearson correlation tests as well as partial correlation analysis. In the case of $\mathrm{p}<0.05$, a statistically significant difference/association was considered.

\section{Results}

The SAT and control groups were similar in terms of age and sex. The WBC count, Neu level, CRP, ESR, and fT3 and fT4 levels were significantly higher in the patient group, whereas the TSH level was significantly lower in the patient group $(\mathrm{p}<0.05)($ Tab. 1$)$.

Evaluation of the patient and control groups in terms of oxidative stress parameters revealed no significant difference between the groups in terms of TAS, TOS, OSI, and PON1 levels. However, TT and NT levels were lower in the SAT group than in the control group $(\mathrm{p}<0.001)$. DS levels were similar. DS/NT and DS/TT levels were significantly higher in the SAT group, whereas NT/TT levels were lower. A comparison of both groups for oxidative stress parameters is presented in Table 2.

In the correlation analysis performed on the whole study population, age was negatively correlated with TT and NT; however, a positive correlation was detected between age and PON1. Furthermore, NT and TT were

Table 1. Demographic characteristics and laboratory findings of the study population

\begin{tabular}{lccc}
\hline & Control $(\mathbf{n}=\mathbf{3 0})$ & Subacute Thyroiditis $(\mathbf{n}=\mathbf{2 5})$ & $\mathbf{p}$ \\
\hline Gender, $\mathrm{n}(\%)$ & & & NS \\
\hline Female & $24(80)$ & $20(80)$ & $5(20)$ \\
\hline Male & $6(20)$ & $38(29-55)$ & NS \\
\hline Age $[$ years] & $35(21-59)$ & $8440(4000-14,000)$ & 0.003 \\
\hline WBC $\left[\mathrm{n} / \mathrm{mm}^{3}\right]$ & $6885(4500-10300)$ & $5280(2510-9760)$ & $<0.001$ \\
\hline Neu $\left[\mathrm{n} / \mathrm{mm}^{3}\right]$ & $3945(2190-6690)$ & $4.5(0.5-13.47)$ & $<0.001$ \\
\hline CRP $[\mathrm{mg} / \mathrm{L}]$ & $0.14(0.01-0.49)$ & $37(9-62)$ & $<0.001$ \\
\hline ESR $[\mathrm{mm} / \mathrm{h}]$ & $11.5(2-29)$ & $5.09(2.96-12.12)$ & 0.001 \\
\hline fT3 $[\mathrm{ng} / \mathrm{dL}]$ & $2.76(2.08-3.85)$ & $2.24(1.07-3.78)$ & $<0.001$ \\
\hline fT4 $[\mathrm{ng} / \mathrm{dL}]$ & $1.1(0.85-1.6)$ & $0.01(0-0.59)$ & $<0.001$ \\
\hline TSH $[\mathrm{mlU} / \mathrm{mL}]$ & $1.82(0.67-3.95)$ & & \\
\hline WBC - white blood cell; Neu - neutrophil; CRP - C-reactive protein; ESR - erythrocyte sedimentation rate; fT3 - free triiodothyronine; fT4 - free thyroxine;
\end{tabular}

Table 2. Oxidative stress parameters of the study population

\begin{tabular}{lccc}
\hline & Control $(\mathbf{n}=\mathbf{3 0})$ & Subacute thyroiditis $(\mathbf{n}=\mathbf{2 5})$ & $\mathbf{p}$ \\
\hline TAS $[\mathrm{mmol}$ Trolox equivalent/L] & $1.54(1.3-2.24)$ & $1.58(1.18-1.88)$ & NS \\
\hline TOS $\left[\mathrm{mmol} / \mathrm{L} \mathrm{H}_{2} \mathrm{O}_{2}\right.$ equivalent/L] & $8.76(3.41-18.43)$ & $8.83(4.91-14.55)$ & NS \\
\hline OSI [arbitrary unit] & $5.9(2.76-11.46)$ & $5.95(2.91-11.3)$ & NS \\
\hline PON1 [U/L] & $292.3(76.5-1019.1)$ & $241.6(105.7-664.3)$ & NS \\
\hline NT $[\mu \mathrm{mol} / \mathrm{L}]$ & $419.45(332.8-522.5)$ & $366(239.4-501.9)$ & $<0.001$ \\
\hline TT $[\mu \mathrm{mol} / \mathrm{L}]$ & $447.5(367.3-549.8)$ & $384.1(258.2-538.3)$ & $<0.001$ \\
\hline DS $[\mu \mathrm{mol} / \mathrm{L}]$ & $15.47(8.25-21.75)$ & $15.45(9.05-28)$ & $\mathrm{NS}$ \\
\hline DS/NT (\%) & $3.6(1.71-5.55)$ & $4.13(2.41-8.59)$ & 0.02 \\
\hline DS/TT $(\%)$ & $3.35(1.66-4.99)$ & $3.82(2.3-7.33)$ & 0.02 \\
\hline NT/TT $(\%)$ & $93.28(90.01-96.69)$ & $92.36(85.34-95.4)$ & 0.02 \\
\hline TAS
\end{tabular}

TAS — total antioxidant status; TOS — total oxidant status; OSI — oxidative stress index; PON1 — paraoxonase 1; NT — native thiol; TT — total thiol; DS — disulphide; NS - nonsignificant 
negatively correlated with WBC count, Neu level, CRP, ESR, and fT4 and positively correlated with TSH level (Tab. 3). The partial correlation analysis performed by adjusting age, WBC count, Neu, ESR, and CRP level revealed a negative correlation between $\mathrm{fT} 3$ and TOS, OSI, DS, DS/NT, and DS/TT and a positive correlation between fT3 and NT/TT (Tab. 3).

A significant and negative association was detected between age and TT level only in the correlation analysis conducted on SAT patients $(\mathrm{r}=-0.442, \mathrm{p}=0.027)$. Furthermore, a negative correlation was found between CRP level and TAS, whereas a positive correlation was detected between CRP level and OSI. There was a negative association between TSH and TAS only in the partial correlation analysis adjusting the age, WBC count, Neu level, CRP, and ESR ( $r=-0.481, \mathrm{p}=0.043)$ (Tab. 3).

\section{Discussion}

We found that NT, TT, and NT/TT values were significantly lower, and DS/NT and DS/TT values were significantly higher, in the SAT group when compared with the control group. Furthermore, we detected significant correlations between thiol levels and inflammatory markers as well as thyroid hormones. We thereby demonstrated a possible association between SAT and oxidative stress.

The aetiopathogenesis of SAT has not been clarified yet. However, it is generally accepted that the disease may present a genetic transition [1]. In particular, the presence of HLA-B35 was detected in two-thirds of the patients, and family series were identified rarely in the literature $[1,12,13]$. Another important determinant is viral factors. It is thought that the disease may develop due to many viral factors such as mumps, rubella, influenza, adenovirus, coxsackie virus, and severe acute respiratory syndrome coronavirus 2 (SARS-CoV-2) lately $[1,14]$. One argument claims that both factors are responsible in the aetiopathogenesis, and the antigens appearing due to viral factors form a complex with HLA-B35 and activate cytotoxic T lymphocytes. Activated T lymphocytes cause damage in thyroid follicle cells, resulting in inflammation [15]. Hernik et al. showed that iron homeostasis and hepcidin also play an important role in the aetiology of SAT [16].

It is known that oxidative stress is related to inflammation and increases in inflammation, and it is also associated with many acute and chronic diseases [4, 9, 17-21]. Previous studies demonstrated that oxidative stress increases in autoimmune thyroid diseases progressing with chronic inflammation such as Hashimoto thyroiditis and Graves' disease, and there may be a cause-and-effect relationship between these diseases and oxidative stress $[4,5,21-23]$. These data suggest the possible association of SAT involving viral factors and immune reactions with inflammation in the pathogenesis with oxidative stress. Furthermore, Moncayo et al. detected lower levels of selenium, which plays a key role in thyroid functions, in patients with SAT [10]. Briefly, the oxidative stress process that appears with the decrease of antioxidant radicals and/or the increase of direct oxidant radicals probably plays a key role in the pathogenesis of SAT.

We did not detect any significant increase in disulphide levels among oxidative stress parameters in SAT patients. However, significantly lower levels of antioxidant parameters (TT and NT) when compared to the control group and significantly higher DS/NT and DS/TT ratios indicate that thiol/disulphide homeostasis is disrupted by oxidative stress. Dynamic thiol/disulphide homeostasis plays a role in many critical mechanisms, and disruption of that balance disrupts some vital cell functions [2, 3, 11, 24]. Organelle pathologies appear due to oxidative stress [5]. Thiols are accepted as essential antioxidant buffers because they have constant interactions with almost all physiological oxidants [20]. One of the major thiol components of the body is glutathione. The glutathione peroxidase enzyme converts glutathione into glutathione disulphide and protects the thyroid gland from oxidative damage by hydrogen peroxide $\left(\mathrm{H}_{2} \mathrm{O}_{2}\right)$, which acts during hormone synthesis [25]. Glutathione peroxidase is a selenium-dependent selenoprotein. Lower selenium levels have already been shown in patients with SAT [10]. Furthermore, it is also known that selenium deficiency increases the virulence of some infectious agents [26]. It is suggested that selenium deficiency may trigger SAT development after viral infection in genetically predisposed individuals. When all these mechanisms and study data are evaluated together, it may be considered that lower thiol levels play a role in the aetiopathogenesis of SAT with a similar mechanism. The main factor may be glutathione in this mechanism. Another finding supporting the hypothesis that dynamic thiol/disulphide homeostasis may play a role in the aetiopathogenesis of SAT is the similarity of other oxidant and antioxidant mechanisms (TAS, TOS, OSI, and PON1); the oxidative imbalance in SAT patients may be caused by inflammation and thyrotoxicosis due to the disease. Thyroid hormones regulate oxidative metabolism and play a role in free radical production [23, 27]. These hormones play a role in the synthesis and degradation of some enzymes including superoxide dismutase, catalase, glutathione peroxidase, and glutathione reductase, as well as some non-enzymatic antioxidants such as vitamins $\mathrm{E}$ and $\mathrm{C}$, glutathione, uric acid, ferritin, transferrin, and ceruloplasmin. The changes in these enzymes 


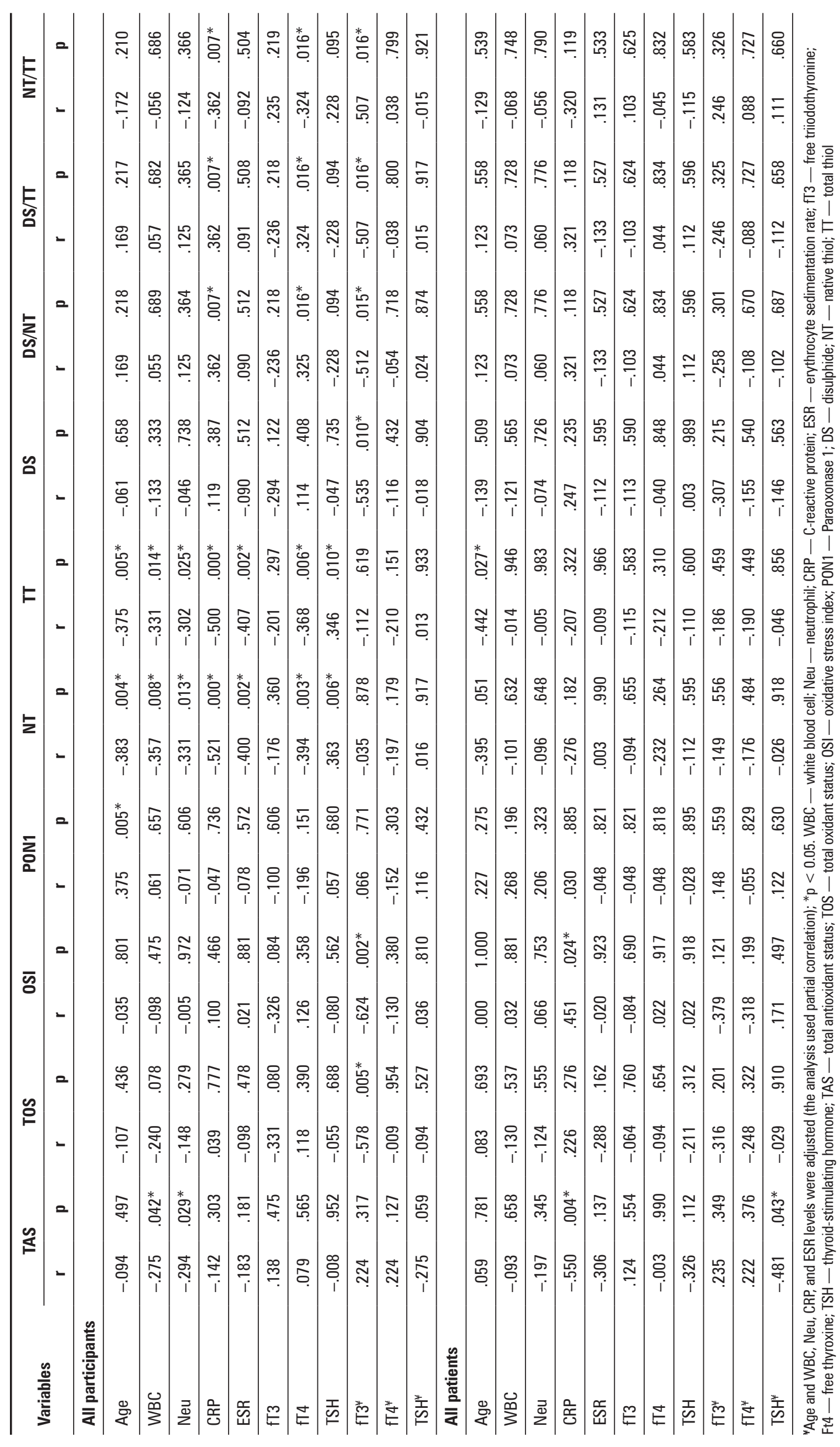


and non-enzymatic substances affect the redox balance of the body. Enzymatic feedback mechanisms thereby regulate thyroid functions. One of the most important effects of thyroid hormones is increasing mitochondrial respiration. This causes up-regulation of reactive oxygen radicals and oxidative damage of membrane lipids. In other words, oxidative stress is known to be associated with thyroid functions both in hypothyroidism and hyperthyroidism [23, 27, 28]. The increase of free radicals along with increased cellular metabolic rate was shown in hyperthyroidism [29, 30]. There are different studies addressing the findings that antioxidant enzymes both increase and decrease $[31,32]$. All patients enrolled in the present study had thyrotoxicosis. However, there was no increase in oxidative parameters (TOS, OSI, and DS). Furthermore, thiol levels, including glutathione, only decreased among antioxidant mechanisms. TAS and PON1 levels were normal, and there was no association between thyroid functions and total and native thiol levels in partial correlation analysis conducted by excluding the inflammation parameters and age in the patients with SAT. These findings suggest that the effect of thyroid hormones on oxidative stress is minimal.

Inflammation increases oxidative stress [4]. The oxidative balance may be disrupted both in chronic and acute inflammation $[4,18]$. However, an increase in levels of oxidative parameters such as TOS and DS is expected in oxidative stress appearing due to inflammation $[4,20]$. In the present study, a negative correlation of NT and TT was detected with WBC count, Neu level, CRP, and ESR level in the whole study population. There was no such association in the patients with SAT. In other words, there was no association between inflammation severity and thiol levels. Maehira et al. showed a negative association between selenium levels and CRP and such a mechanism appears for NF- $\kappa \beta$ [33]. A negative association was also shown between selenium levels and IL-6 [34]. These data reveal that lower thiol levels might be associated with an increase in inflammation markers and inflammation in patients with SAT. The expected inflammation-induced increase in oxidant parameters may not always be detected because the patients enrolled in the study were diagnosed early. Reversible thiol/disulphide homoeostasis is the earliest stage of protein oxidation, and antioxidant parameters may start to decrease first with such a balance [5].

In consideration of all data obtained in the present study as well as the information in the literature, we believe that the dynamic thiol/disulphide balance may play a role in the aetiopathogenesis of SAT. However, we are aware of the necessity of other data to support this claim. The present study has some limitations at this stage. The most important limitation of our study is its cross-sectional design. After complete recovery of inflammation and thyrotoxicosis findings, re-evaluation of oxidative stress parameters would provide a better understanding. Another limitation is the lack of glutathione, glutathione peroxidase, and selenium analyses. Furthermore, viral factors, HLA, and thyroid antibodies, which may act in the aetiopathogenesis of SAT, were not evaluated.

\section{Conclusions}

The present study has presented for the first time that there may be an association between SAT and oxidative stress; thiol levels are significantly decreased and thiol/disulphide homoeostasis is disrupted in patients with SAT. The possible benefits of antioxidant therapies for the treatment and prevention of the disease and recurrence in patients with SAT should be considered. Large-scale studies are still required to reveal this association exactly.

\section{Conflict of interest}

The authors declare that they have no conflict of interest.

\section{Funding}

This research did not receive any specific grant from funding agencies in the public, commercial, or not-for-profit sectors.

\section{References}

1. Shrestha RT, Hennessey J. Acute and Subacute, and Riedel's Thyroiditis. Endotext, MDText.com Inc 2015.

2. Ates I, Ozkayar N, Topcuoglu C, et al. Relationship between oxidative stress parameters and asymptomatic organ damage in hypertensive patients without diabetes mellitus. Scand Cardiovasc J. 2015; 49(5): 249-256, doi: 10.3109/14017431.2015.1060355, indexed in Pubmed: 26053412.

3. Villanueva I, Alva-Sánchez C, Pacheco-Rosado J. The role of thyroid hormones as inductors of oxidative stress and neurodegeneration. Oxid Med Cell Longev. 2013; 2013: 218145, doi: 10.1155/2013/218145, indexed in Pubmed: 24386502

4. Ates I, Yilmaz FM, Altay M, et al. The relationship between oxidative stress and autoimmunity in Hashimoto's thyroiditis. Eur J Endocrinol. 2015; 173(6): 791-799, doi: 10.1530/EJE-15-0617, indexed in Pubmed: 26340971

5. Ates I, Altay M, Yilmaz FM, et al. Dynamic thiol/disulfide homeostasis in patients with autoimmune subclinical hypothyroidism. Endocr Res. 2016; 41(4): 343-349, doi: 10.3109/07435800.2016.1156124, indexed in Pubmed: 27045442

6. Finkel T. Redox-dependent signal transduction. FEBS Lett. 2000; 476(1-2) 52-54, doi: 10.1016/s0014-5793(00)01669-0, indexed in Pubmed: 10878249.

7. Dean RT, Fu S, Stocker R, et al. Biochemistry and pathology of radical-mediated protein oxidation. Biochem J. 1997; 324 ( Pt 1): 1-18, doi: 10.1042/bj3240001, indexed in Pubmed: 9164834.

8. Jones DP, Liang Y. Measuring the poise of thiol/disulfide couples in vivo. Free Radic Biol Med. 2009; 47(10): 1329-1338, doi: 10.1016/j.freeradbiomed.2009.08.021, indexed in Pubmed: 19715755.

9. Ates I, Ozkayar N, Altay M, et al. Is disulphide/thiol ratio related to blood pressure in masked hypertension? Clin Exp Hypertens. 2016; 38(2): 150-154, doi: 10.3109/10641963.2015.1060995, indexed in Pubmed: 26418425

10. Moncayo R, Kroiss A, Oberwinkler M, et al. The role of selenium, vitamin $C$, and zinc in benign thyroid diseases and of selenium in malignant thyroid diseases: Low selenium levels are found in subacute and silent thyroiditis and in papillary and follicular carcinoma. 
BMC Endocr Disord. 2008; 8: 2, doi: 10.1186/1472-6823-8-2, indexed in Pubmed: 18221503

11. Erel O, Neselioglu S. A novel and automated assay for thiol/disulphide homeostasis. Clin Biochem. 2014; 47(18): 326-332, doi: 10.1016/j.clinbiochem.2014.09.026, indexed in Pubmed: 25304913.

12. Buc M, Nyulassy S, Hnilica P, et al. HLA-BW35 and subacute de Quervain's thyroiditis [proceedings]. Diabete Metab. 1976; 2(3): 163-274, indexed in Pubmed: 1010141

13. Kramer AB, Roozendaal C, Dullaart RPF. Familial occurrence of subacute thyroiditis associated with human leukocyte antigen-B35. Thyroid. 2004; 14(7): 544-547, doi: 10.1089/1050725041517048, indexed in Pubmed: 15307945.

14. Stasiak M, Lewiński A. New aspects in the pathogenesis and management of subacute thyroiditis. Rev Endocr Metab Disord. 2021 [Epub ahead of print], doi: 10.1007/s11154-021-09648-y, indexed in Pubmed: 33950404.

15. Burman KD. Subacute thyroiditis Up To Date. https://www.uptodate.com/contents/subacute-thyroiditis?search $=$ subacute $\% 20$ thyroiditis\&source $=$ search_result\&selected Title $=1 \sim 38 \&$ usage type $=$ default\&display_rank $=12019$.

16. Hernik A, Szczepanek-Parulska E, Filipowicz D, et al. Hepcidin and Iron Homeostasis in Patients with Subacute Thyroiditis and Healthy Subjects. Mediators Inflamm. 2019; 2019: 5764061, doi: 10.1155/2019/5764061, indexed in Pubmed: 30936776.

17. Matteucci E, Giampietro O. Thiol signalling network with an eye to diabetes. Molecules. 2010; 15(12): 8890-8903, doi: 10.3390/molecules15128890, indexed in Pubmed: 21135801.

18. Go YM, Jones DP. Cysteine/cystine redox signaling in cardiovascular disease. Free Radic Biol Med. 2011; 50(4): 495-509, doi: 10.1016/j.freeradbiomed.2010.11.029, indexed in Pubmed: 21130865.

19. Prabhu A, Sarcar B, Kahali S, et al. Cysteine catabolism: a novel metabolic pathway contributing to glioblastoma growth. Cancer Res. 2014; 74(3): 787-796, doi: 10.1158/0008-5472.CAN-13-1423, indexed in Pubmed: 24351290.

20. Köseoğlu H, Alısıı M, Başaran M, et al. Dynamic thiol/disulphide homeostasis in acute pancreatitis. Turk J Gastroenterol. 2018; 29(3): 348-353, doi: 10.5152/tjg.2018.17499, indexed in Pubmed: 29755020.

21. Ates I, Arikan MF, Altay M, et al. The effect of oxidative stress on the progression of Hashimoto's thyroiditis. Arch Physiol Biochem. 2018; 124(4): 351-356, doi: 10.1080/13813455.2017.1408660, indexed in Pubmed: 29185364.

22. Santi A, Duarte MM, Moresco RN, et al. Association between thyroid hormones, lipids and oxidative stress biomarkers in overt hypothyroidism. Clin Chem Lab Med. 2010; 48(11): 1635-1639, doi: 10.1515/CCLM.2010.309, indexed in Pubmed: 20704527.
23. Wang D, Feng JF, Zeng P, et al. Total oxidant/antioxidant status in sera of patients with thyroid cancers. Endocr Relat Cancer. 2011; 18(6): 773-782, doi: 10.1530/ERC-11-0230, indexed in Pubmed: 22002574.

24. Biswas S, Chida AS, Rahman I. Redox modifications of protein-thiols: emerging roles in cell signaling. Biochem Pharmacol. 2006; 71(5): 551-564, doi: 10.1016/j.bcp.2005.10.044, indexed in Pubmed: 16337153.

25. Rostami R, Aghasi MR, Mohammadi A, et al. Enhanced oxidative stress in Hashimoto's thyroiditis: inter-relationships to biomarkers of thyroid function. Clin Biochem. 2013; 46(4-5): 308-312, doi: 10.1016/j. clinbiochem.2012.11.021, indexed in Pubmed: 23219737.

26. Xie Bo, Zhou JF, Lu Q, et al. Oxidative stress in patients with acute coxsackie virus myocarditis. Biomed Environ Sci. 2002; 15(1): 48-57, indexed in Pubmed: 12046548.

27. Erdamar $\mathrm{H}$, Demirci $\mathrm{H}$, Yaman $\mathrm{H}$, et al. The effect of hypothyroidism hyperthyroidism, and their treatment on parameters of oxidative stress and antioxidant status. Clin Chem Lab Med. 2008; 46(7): 1004-1010, doi: 10.1515/CCLM.2008.183, indexed in Pubmed: 18605962.

28. Resch U, Helsel G, Tatzber F, et al. Antioxidant status in thyroid dysfunction. Clin Chem Lab Med. 2002; 40(11): 1132-1134, doi: 10.1515/CCLM.2002.198, indexed in Pubmed: 12521231

29. Chance B, Sies H, Boveris A. Hydroperoxide metabolism in mammalian organs. Physiol Rev. 1979; 59(3): 527-605, doi: 10.1152/physrev.1979.59.3.527, indexed in Pubmed: 37532.

30. Castilho RF, Kowaltowski AJ, Vercesi AE 3,5,3'-triiodothyronine induces mitochondrial permeability transition mediated by reactive oxygen species and membrane protein thiol oxidation. Arch Biochem Biophys. 1998; 354(1): 151-157, doi: 10.1006/abbi.1998.0657, indexed in Pubmed: 9633610.

31. Asayama K, Dobashi K, Hayashibe H, et al. Lipid peroxidation and free radical scavengers in thyroid dysfunction in the rat: a possible mechanism of injury to heart and skeletal muscle in hyperthyroidism. Endocrinology. 1987; 121(6): 2112-2118, doi: 10.1210/endo-121-6-2112, indexed in Pubmed: 2824181.

32. Morini P, Casalino E, Sblano C, et al. The response of rat liver lipid peroxidation, antioxidant enzyme activities and glutathione concentration to the thyroid hormone. Int J Biochem. 1991; 23(10): 1025-1030, doi: 10.1016/0020-711x(91)90140-i, indexed in Pubmed: 1786846.

33. Maehira F, Miyagi I, Eguchi Y. Selenium regulates transcription factor NF-kappaB activation during the acute phase reaction. Clin Chim Acta. 2003; 334(1-2): 163-171, doi: 10.1016/s0009-8981(03)00223-7, indexed in Pubmed: 12867288.

34. Sakr Y, Reinhart K, Bloos F, et al. Time course and relationship between plasma selenium concentrations, systemic inflammatory response, sepsis, and multiorgan failure. Br J Anaesth. 2007; 98(6): 775-784, doi: 10.1093/bja/aem091, indexed in Pubmed: 17478454 